\title{
Intergenerational Transmission of Internalizing Behavior: The Role of Maternal Psychopathology, Child Responsiveness and Maternal Attachment Style Insecurity
}

\author{
Corinna Reck ${ }^{\mathrm{a}}$ Nora Nonnenmacher ${ }^{\mathrm{b}}$ Anna-Lena Zietlow ${ }^{\mathrm{b}}$ \\ a Department of Psychology, Ludwig Maximilians University Munich, Munich, and b Heidelberg University Hospital, \\ Heidelberg, Germany
}

\section{Key Words}

Child internalizing behavior - Child responsiveness .

Depression · Anxiety disorders · Maternal attachment insecurity

\begin{abstract}
Background: Maternal depression and anxiety disorders are risk factors for the development of internalizing disorders in offspring. Maternal attachment has been discussed as one factor accounting for transmission. The aim of this study was to investigate child internalizing behavior at preschool age on a symptomatic and behavioral level and possible links to maternal mental health over time and maternal attachment style insecurity in a sample of postpartum depressed and anxious mothers. Sampling and Methods: Child internalizing behavior at preschool age was rated by the Child Behavior Checklist (CBCL), the Caregiver-Teacher Report Form (CTRF), and during a mother-child free-play situation. We focused on child responsiveness as it has been linked to child internalizing behavior. Maternal attachment style insecurity was tested to mediate the link between maternal mental
\end{abstract}

health (assessed postpartum and at preschool age with the Structured Clinical Interview for DSM-IV Axis-I Disorders, SCID-I) and child internalizing behavior/child responsiveness. Of the overall sample $(n=58), 28$ women were diagnosed with postpartum depression and/or anxiety disorders according to DSM-IV, and 30 were healthy controls. Data were collected 3-9 months after delivery and at preschool age (mean $=4.6$ years). Results: At preschool age, children of postpartum depressed and anxious mothers were rated significantly higher on child internalizing behavior by mothers, fathers, and additional caregivers compared to the control group. Child internalizing behavior rated by mothers was influenced by current psychiatric symptoms; maternal attachment style insecurity did not mediate this link. During interaction, children in the clinical group displayed significantly less child responsiveness compared to the control group. Maternal attachment style insecurity mediated the relationship between maternal mental health over time and child responsiveness. Conclusions: The results emphasize the need for interventions focusing on mother-child interaction and maternal attachment.

(c) 2016 S. Karger AG, Basel

\section{KARGER}

E-Mail karger@karger.com www.karger.com/psp
(C) 2016 S. Karger AG, Basel

0254-4962/16/0494-0277\$39.50/0
Prof. Dr. Corinna Reck

Department of Psychology

Ludwig Maximilians University Munich

Leopoldstraße 13, DE-80802 Munich (Germany)

E-Mail Corinna.Reck@psy.lmu.de 


\section{Introduction}

The risk for the development of internalizing symptoms is raised in children of mothers with depression and anxiety disorders [1]. Internalizing problem behaviors, which can be seen as a precursor for later affective psychopathology, include emotional reactive behavior, anxious/depressive symptoms, somatic complaints, and withdrawn behavior [2]. This is also expressed during the mother-child interaction in the responsiveness a child displays to the caregiver, characterized by less emotional and social response to and ignorance of the overture of the caregiver, answering in an unenthusiastic way or appearing bland, sometimes even shy, whiny, anxious, complaining, or withdrawn [3]. Associations between the child's emotional responsiveness to the mother and internalizing behavior have been reported [4].

The most common postpartum psychiatric disorders in Germany are depression, with prevalence rates of $6.1 \%$, and anxiety disorders, with prevalence rates of $11.1 \%$ [5]. Co-occurring anxiety and depressive symptoms are frequent and related to severity [6]. The onset, severity, and chronicity of maternal affective disorders are considered to influence the child's socioemotional development $[7,8]$.

There is strong evidence for intergenerational transmission of depression and anxiety disorders, with an elevation of up to $4-6$ times $[9,10]$, especially if the mother as primary caregiver is affected [for review, see 10,11]. Multiple factors for intergenerational transmission have been discussed: the child's adaption of maternal affective states [12,13], impaired parenting behaviors [14], genetics [15], and the quality of the mother-child interaction [13].

It is well documented that interactions with the primary caregiver exert a strong influence on child development [for review, see 16]. Interaction behavior of depressed mothers is characterized by less sensitivity, more negative affect, and withdrawal or intrusiveness [17]. Results of interaction studies focusing on postpartum anxiety disorders are more heterogeneous regarding reduced maternal sensitivity [18], but these mothers have been found to interact less positively and more intrusively than controls [19]. The mother-child interaction is understood as a mutual process in which mother and child as active partners adjust to the behavior of the other [13]. As mothers with affective disorders are often impaired regarding sensitivity [20], children may not receive sufficient support for their affect regulation [17], leading to reduced competencies regarding tolerance of negative affect and stress regulation [13]. As a consequence of the lacking emotional support, children display less positive affect, less social activity [12], less responsiveness, and less involvement during interactions [20].

Mothers with reduced maternal sensitivity are often insecurely attached [21], and an insecure attachment increases the risk for maternal depression and anxiety disorders [22, 23]. Maternal attachment can therefore be seen as another risk factor for intergenerational transmission. Research suggests that attachment patterns often transmit from mothers to offspring [for review, see 24] by verbal and nonverbal interactions [21]. Maternal insecure attachment is associated with lower observed child responsiveness [25] and higher internalizing behavior reported by the mother [26].

Most studies use parental self-report questionnaires for the assessment of internalizing child behavior, and the results indicate an overestimation of internalizing symptoms by women with affective disorders compared to healthy controls or additional caregivers [27]. One explanation could be that affected women are more sensitive to the core symptoms of these disorders [27]. In contrast, 'cognitive distortion hypothesis' assumes that due to psychiatric symptoms these women interpret other people and their behaviors more negatively than healthy individuals [28], leading to more negative ratings of child behavior [for review, see 29].

The aim of this study was to investigate reported and observed internalizing child behavior at preschool age in the light of postpartum depression and anxiety disorders, taking the development of maternal mental health into account. We assumed that children of the clinical group would be rated higher on internalizing behavior than children of the control group by mothers, fathers, and additional caregivers. Furthermore, we hypothesized that children of the clinical group interact less responsively during interaction with their mothers. As depression and anxiety disorders often negatively affect the mother-child interaction (expressed in lower maternal sensitivity), both are associated with maternal attachment style insecurity. Therefore, we hypothesized that maternal attachment style insecurity would mediate the relationship between maternal mental health and the reported as well as observed child internalizing behavior.

\section{Methods}

Procedure and Participants

Data were collected from 2004 to 2011 in two middle-sized towns in Southern Germany. Subjects were recruited in local maternity hospitals and from a mother-infant unit. The screening 
procedure is described elsewhere in detail [5]. The German version of the Structured Clinical Interview for DSM-IV Axis-I Disorders (SCID-I; [30]) was completed at two assessments: the first $\left(\mathrm{T}_{1}\right)$ within 9 months after delivery, and the second $\left(T_{2}\right) 3-6$ years later (mean $=4.6$ years). At $\mathrm{T}_{2}$ child internalizing behavior was assessed by the German versions of the Child Behavior Checklist (CBCL 1.5-5), and the Caregiver-Teacher Report Form (C-TRF) 1.5-5 $[31,32]$ rated by mothers, fathers, and additional caregivers and during a 15-min free-play mother-child interaction. Additional caregivers were defined as day care providers, such as kindergarten teachers and day care parents who were involved in child rearing. Furthermore, maternal attachment style insecurity was assessed with the Vulnerable Attachment Style Questionnaire (VASQ) [33]. The mother-child interaction was coded by two trained and reliable psychologists who were blinded to the mental health status of the mothers using the Emotional Availability Scales (EA Scales) [3]. In order to assure reliability, $30 \%$ of the videos $(n=19)$ were recorded by a second independent observer. Cohen's kappa resulted in $\kappa=0.78$ for child responsiveness.

The initial sample size $(n=66)$ was reduced to 58 due to incomplete follow-up questionnaires of the participants. Women were allocated to the corresponding group due to their postpartum mental health status assessed with the SCID-I at $\mathrm{T}_{1}$. All women in the clinical group fulfilled the criterion of the additional code 'postpartum' according to DSM-IV with an onset within 4 weeks after delivery. Women with one or more of the following diagnoses at $\mathrm{T}_{1}$ were assigned to the clinical group $(\mathrm{n}=28)$ : major depression, generalized anxiety disorder, agoraphobia, panic disorder, and social phobia. In the clinical sample, $23(82.1 \%)$ were inpatients of a mother-infant unit, $3(10.7 \%)$ were outpatients, and 2 mothers $(7.1 \%)$ were not in psychotherapeutic treatment. At $\mathrm{T}_{2}, 13$ women (46.4\%) fulfilled the SCID criteria for depression and anxiety disorders, and 4 women (14.3\%) reported partly remitted symptoms. In this group, 11 women (39.3\%) had diagnoses solely in the postpartum period $\left(\mathrm{T}_{1}\right) ; 13$ women $(46.4 \%)$ at $\mathrm{T}_{1}$ and 9 women $(32.1 \%)$ at $\mathrm{T}_{2}$ had concurrent depression and anxiety disorders. The control group was comprised of 30 women without current or a history of psychiatric disorders or psychotherapy. All participating mothers needed to be at least 18 years old and have adequate knowledge of the German language. All participating children were born full term. The study protocol was approved by the independent Ethics Committee of the Medical Faculty of Heidelberg University Hospital. Written informed consent was obtained from every participating woman after the study procedures had been fully explained.

\section{Sample Characteristics}

At $\mathrm{T}_{1}$ maternal age ranged from 26 to 46 years $(\mathrm{mean}=37.41$ years, $\mathrm{SD}=4.27)$ within the overall sample $(\mathrm{n}=58)$. On average women had 2.14 children $(\mathrm{SD}=1.02$, range: $1-6)$. At $\mathrm{T}_{2}$ the age of the children ranged from 3.01 to 6.00 years (mean $=4.61, \mathrm{SD}=$ 0.71 ). Of the total sample, 31 children (53.4\%) were male. Maternal educational level in the overall sample was distributed as follows: $16(27.6 \%)$ completed intermediate secondary education, 14 (24.1\%) qualified for university entrance, and 28 (48.3\%) held a university degree.

\section{Statistical Analyses}

The Statistical Package for Social Sciences (IBM ${ }^{\mathrm{TM}}$ SPSS $^{\circledR}$ v. 22.0) was used for all analyses. To ensure comparability between both groups, differences concerning maternal age, child age, number of children, maternal education, and child gender were explored using t tests and $\chi^{2}$ tests before carrying out the main analyses. $t$ tests were conducted to test for group differences regarding self-reported internalizing behavior (mother, father, and additional caregiver) and observed child responsiveness during the mother-child interaction. Due to directed hypotheses, one-tailed testing was performed.

As previous studies stress the importance of current psychiatric diagnoses on the maternal perception of the relationship to the child [34] and for further determination of specific characteristics of maternal psychopathology we operationalized a variable 'maternal mental health over time' for the assessment from postpartum up to preschool age containing three conditions: without a history of SCID diagnoses (never), diagnoses postpartum and not at $\mathrm{T}_{2}$ (not current), and partly remitted symptoms or current diagnoses at $\mathrm{T}_{2}$ (partly remitted/current). As we focused on intergenerational transmission from mother to child, only maternal ratings of child internalizing behavior and observed mother-child interaction were included in the subsequent analyses.

Pearson's correlations were conducted to test for associations between child internalizing behavior (CBCL), observed child responsiveness (EA Scales), maternal mental health (postpartum and over time), and maternal attachment style insecurity (insecurity and proximity seeking). As the proximity seeking scale did not correlate to reported and observed child responsiveness only insecurity was taken into account. Two mediation analyses were conducted, hypothesizing that maternal attachment style insecurity would mediate the link between maternal mental health over time and internalizing child behavior (CBCL) as well as observed child responsiveness (EA Scales). Post hoc tests (Tukey) were conducted to test for subgroup differences regarding maternal mental health over time.

\section{Measures}

German Versions of the CBCL 1.5-5/C-TRF 1.5-5

These CBCL/C-TRF $[31,32]$ assess internalizing and externalizing behavior problems in children $(0=$ absent, $1=$ occurs sometimes, 2 = occurs often). Internalizing behavior includes emotional reactive behavior, anxious/depressive symptoms, somatic complaints, and withdrawn behavior, whereas attention deficits and aggressive behavior are characteristics for externalizing behavior. In both questionnaires higher scores indicate more problematic child behavior. T-scores $<60$ can be seen as clinically relevant. Psychometric properties are described as satisfying.

\section{Vulnerable Attachment Style Questionnaire}

The VASQ [33] is a self-report questionnaire measuring behaviors, feelings, and attitudes regarding adult attachment styles. 22 five-point Likert-scaled items are assigned to two scales: insecurity and proximity seeking, both related to insecure attachment. Insecurity is closely related to avoidant attachment and proximity seeking to enmeshed attachment. Higher scores indicate higher attachment insecurity. Psychometric properties are satisfying [33].

Structured Clinical Interview for DSM-IV Axis I Disorders

The German SCID-I [30] is a widely used semistructured interview for the diagnosis of selected Axis-I mental disorders accord- 
Table 1. Sample characteristics $(\mathrm{n}=58)$

\begin{tabular}{|c|c|c|c|c|c|}
\hline & \multicolumn{2}{|c|}{ Control group $(\mathrm{n}=30)$} & \multicolumn{2}{|c|}{ Clinical group $(\mathrm{n}=28)$} & \multirow[t]{2}{*}{$\mathrm{p}$} \\
\hline & mean $\pm \mathrm{SD}$ & range & mean $\pm \mathrm{SD}$ & range & \\
\hline Maternal age $T_{1}$, years & $37.83 \pm 4.65$ & $26-46$ & $36.96 \pm 3.84$ & $30-45$ & $0.44^{\mathrm{a}}$ \\
\hline Number of children & $1.93 \pm 0.69$ & $1-4$ & $2.38 \pm 1.27$ & $1-6$ & $0.11^{\mathrm{a}}$ \\
\hline Child age $\mathrm{T}_{2}$, years & $4.63 \pm 0.84$ & $3.01-6.00$ & $4.59 \pm 0.57$ & $3.04-5.11$ & $0.80^{\mathrm{a}}$ \\
\hline Child gender (male) & $21(70.0 \%)$ & & $10(35.7 \%)$ & & $0.02^{\mathrm{b} *}$ \\
\hline Education & & & & & $0.94^{\mathrm{b}}$ \\
\hline Secondary modern school & $7(23.3 \%)$ & & $9(32.1 \%)$ & & \\
\hline University entrance qualification & $7(23.4 \%)$ & & $7(25.0 \%)$ & & \\
\hline University degree & $16(53.3 \%)$ & & $12(42.9 \%)$ & & \\
\hline \multicolumn{6}{|l|}{ SCID-I diagnoses over time } \\
\hline Never & $30(51.7 \%)$ & & & & \\
\hline No current diagnosis & & & $11(39.3 \%)$ & & \\
\hline Partly remitted/current diagnosis & & & $17(60.7 \%)$ & & \\
\hline
\end{tabular}

${ }^{*} \mathrm{p}<0.01 .^{\mathrm{a}} \mathrm{t}$ test. ${ }^{\mathrm{b}} \chi^{2}$ test. Values for child gender (male), education and SCID-I diagnoses over time indicate absolute frequency.

ing of DSM-IV. The authors of the German version evaluated satisfying psychometric properties regarding reliability, applicability, and efficacy.

Emotional Availability Scales

This coding system [3] assesses the emotional quality of the mother-child interaction and is rated on six dimensions, four addressing maternal and two child behavior. In this study only child responsiveness, describing the degree to which a child is responsive to the adult and enjoys the interaction but at the same time balances with autonomous activities, was included in the analyses. Higher scores indicate better responsiveness towards the mother.

Demographic Information Form and Informed Consent

The demographic information form included data about number and age of children, maternal education, and age and was filled out by every participating woman.

\section{Results}

\section{Preliminary Data Analyses}

Analyses regarding comparability between the clinical and the control group revealed no significant differences except for child gender. As it was not associated with child internalizing behavior in a one-way ANOVA, we desisted from controlling child gender in further statistical analyses. Sample characteristics are shown in table 1.

\section{Main Analyses}

Reported and Observed Internalizing Child Behavior

Mothers, fathers, and additional caregivers reported significantly higher rates of internalizing behavior in children of the clinical group. CBCL T-scores are demonstrated in table 2; all mean scores were below the clinical cutoff (>60). Regarding observed child responsiveness, children in the clinical group displayed significantly less responsive behavior during the mother-child interaction.

Mediation Analyses Regarding Maternal Attachment Style Insecurity

Before carrying out mediation analyses, Pearson's correlations between maternal mental health in the postpartum period and over time, maternal attachment style insecurity (insecurity, proximity seeking), and mothers' ratings of child internalizing behavior (CBCL) and observed child responsiveness (EA Scales) were conducted; these are illustrated in table 3 .

Regarding child internalizing behavior reported by the mother, the first mediation analysis validated the effect of maternal mental health over time on child internalizing behavior (model $\mathrm{I}, \beta=0.442, \mathrm{t}=3.683, \mathrm{p}=0.001$ ) and on maternal attachment style insecurity (model II, $\beta=0.563$, $\mathrm{t}=5.094, \mathrm{p}<0.001$ ). Including both predictors, only maternal mental health over time $(\beta=0.302, t=2.120, \mathrm{p}=$ 0.039 ) significantly contributed to the model with a medium effect. Maternal attachment style insecurity did not 
Table 2. One-tailed group comparisons of rated and observed internalizing child behavior

\begin{tabular}{|c|c|c|c|c|c|c|c|c|}
\hline & Group & $\mathrm{n}$ & Mean & SE & SD & $\mathrm{F}$ & $\mathrm{t}$ & $\mathrm{p}$ \\
\hline \multirow[t]{2}{*}{ Maternal rating (CBCL) } & control & 30 & 43.53 & 1.77 & 9.71 & \multirow{2}{*}{2.973} & \multirow{2}{*}{3.063} & \multirow{2}{*}{0.003} \\
\hline & clinical & 28 & 52.14 & 2.35 & 12.44 & & & \\
\hline \multirow[t]{2}{*}{ Paternal rating (CBCL) } & control & 21 & 40.76 & 1.61 & 7.36 & \multirow{2}{*}{7.705} & \multirow{2}{*}{2.403} & \multirow{2}{*}{0.011} \\
\hline & clinical & 20 & 48.95 & 2.95 & 13.18 & & & \\
\hline \multirow[t]{2}{*}{ Caregiver rating (C-TRF) } & control & 18 & 46.33 & 2.06 & 8.72 & \multirow{2}{*}{1.294} & \multirow{2}{*}{2.004} & \multirow{2}{*}{0.028} \\
\hline & clinical & 19 & 51.26 & 1.43 & 6.23 & & & \\
\hline \multirow[t]{2}{*}{ Child responsiveness (EA Scales) } & control & 29 & 4.37 & 0.22 & 1.18 & \multirow{2}{*}{3.646} & \multirow{2}{*}{-3.538} & \multirow{2}{*}{0.001} \\
\hline & clinical & 25 & 3.40 & 0.17 & 0.87 & & & \\
\hline
\end{tabular}

Table 3. Pearson's correlations between maternal mental health, rated and observed child internalizing behavior and maternal attachment style insecurity $(\mathrm{n}=58)$

\begin{tabular}{lcccccc}
\hline & $\begin{array}{l}\text { Mental health } \\
\text { postpartum }\end{array}$ & $\begin{array}{l}\text { Mental health } \\
\text { over time }\end{array}$ & $\begin{array}{l}\text { Child internal- } \\
\text { izing behavior }\end{array}$ & $\begin{array}{l}\text { Child } \\
\text { responsiveness }\end{array}$ & $\begin{array}{l}\text { Maternal attach- } \\
\text { ment insecurity }\end{array}$ & $\begin{array}{l}\text { Maternal } \\
\text { proximity seeking }\end{array}$ \\
\hline Mental health postpartum & $/$ & $-0.921^{* *}$ & $-0.367^{* *}$ & $0.435^{* *}$ & $-0.550^{* *}$ & -0.192 \\
Mental health over time & $-0.921^{* *}$ & $/$ & $0.442^{* *}$ & $-0.391^{* *}$ & $0.563^{* *}$ & 0.238 \\
Child internalizing behavior & $-0.367^{* *}$ & $0.442^{* *}$ & $/$ & $-0.363^{* *}$ & $0.418^{* *}$ & 0.104 \\
Child responsiveness & $0.435^{* *}$ & $-0.391^{* *}$ & $-0.363^{* *}$ & $/$ & $-0.442^{* *}$ & -0.105 \\
Maternal attachment insecurity & $-0.550^{* *}$ & $0.563^{* *}$ & $0.418^{* *}$ & $-0.442^{* *}$ & $/$ & $0.346^{* *}$ \\
Maternal proximity seeking & -0.192 & 0.238 & 0.104 & -0.105 & $0.346^{* *}$ & $/$ \\
\hline
\end{tabular}

${ }^{a}$ Maternal rating of child internalizing behavior. ${ }^{* *} \mathrm{p}<0.001$.

significantly contribute to the model anymore as it reached only marginal significance $(\beta=0.248, t=1.739$, $\mathrm{p}=0.088)$. Therefore, maternal attachment style insecurity did not mediate. Multicollinearity seemed unlikely as the variance inflation factor, assessing the impact of collinearity among the variables, had a value of $\approx 1.46$. Post hoc tests (Tukey) revealed that women with current or partly remitted SCID diagnoses differed significantly in their ratings of internalizing child behavior from women who never had diagnoses $(\Delta$ mean $=12.23, \mathrm{SE}=3.26, \mathrm{p}=$ 0.001 ) and only marginally from women without current diagnoses $(\Delta$ mean $=9.22, \mathrm{SE}=4.16, \mathrm{p}=0.077)$. Women who never had diagnoses and women with no current diagnoses did not differ significantly $(\Delta$ mean $=3.01, \mathrm{SE}=$ $3.79, \mathrm{p}=0.707)$.

Regarding observed child responsiveness during the mother-child interaction, mediation analysis validated the effect of maternal mental health over time on child responsiveness ( model I, $\beta=-0.391, t=-3.065, p=0.003$ ) and on maternal attachment style insecurity (model II, $\beta=0.563, t=5.094, p<0.001$ ). Including both predictors in the regression analysis, the effect of mental health over

Transmission of Internalizing Child Behavior time on child responsiveness did not reach significance $(\beta=-0.208, t=-1.396, p=0.169)$, and therefore maternal attachment style insecurity $(\beta=-0.325, \mathrm{t}=-2.182, \mathrm{p}=$ 0.034 ) was validated as a mediator. As the variance inflation factor was $\approx 1.46$, multicollinearity seemed unlikely. Post hoc tests (Tukey) revealed that children of mothers who never had SCID diagnoses differed significantly from children of mothers with current or partly remitted SCID diagnoses $(\Delta$ mean $=0.97, \mathrm{SE}=0.34, \mathrm{p}=0.019)$ and from children of mothers with no current diagnoses $(\Delta$ mean $=1.03, \mathrm{SE}=0.38, \mathrm{p}=0.022)$ in their responsiveness during interaction. Children of women with current or partly remitted diagnoses and children of women with no current diagnoses did not differ significantly $(\Delta$ mean $=0.065, \mathrm{SE}=0.43, \mathrm{p}=0.987)$.

\section{Discussion}

The aim of this study was to investigate child internalizing behavior at preschool age rated with a multimethod perspective on a symptomatic and behavioral level in the 
light of postpartum depression and anxiety disorders. In addition, the impact of maternal mental health over time and maternal attachment insecurity were taken into account. Mothers, fathers, and additional caregivers reported more internalizing behavior in children of the clinical group compared to healthy controls. As not only maternal but also paternal and caregiver ratings indicated higher internalizing symptoms, it can be assumed that these children indeed show more internalizing symptoms. Although internalizing behavior was reported not to be clinically relevant, $\mathrm{T}$-scores of all raters were allocated in the medium range and did not differ on a descriptive level. Therefore, our data did not support cognitive distortion hypothesis, which is in line with studies reporting no differences in ratings of mothers with affective disorders and additional caregivers [27]. Nevertheless, the results underline that postpartum mood disorders can negatively influence the child's socioemotional development up to preschool age.

The higher rates of reported internalizing behavior were validated by observed child responsiveness. Children of the clinical group interacted less responsively with their mothers. Furthermore, internalizing child behavior reported by mothers correlated significantly with child responsiveness during interaction, indicating that maternal perception of child internalizing behavior can be linked to observational measures [4]. Our results underline that the CBCL is a valid and cost-effective measure to screen for child internalizing behavior. Conversely, child behavior during the mother-child interaction can be seen as a predictor for internalizing behavior already generalized to other environments, as the CBCL not only assesses behavioral and socioemotional functioning in the family but also in different social contexts. This suggests that a history of interaction experiences with the mother influences the child's expectations and responses to the social world.

Contradictory to our hypothesis regarding maternal attachment style insecurity mediating the relationship between maternal mental health over time and the reported as well as observed internalizing child behavior, only the latter was mediated. Although, maternal attachment style insecurity was linked to the maternal perception of child internalizing behavior, when additionally taking maternal mental health over time into account, attachment style insecurity reached only marginal significance. Significant positive correlations indicated that mothers who are more affected by psychiatric symptoms report higher attachment insecurity, which is in line with previous research $[22,23]$. As the results suggest that maternal mental health over time accounted for the effect on reported child internalizing behaviors, future studies should investigate this relationship in more detail in larger samples.

Post hoc tests highlighted the importance of current or partly remitted affective symptoms, as children of currently affected women were rated significantly higher on internalizing behavior compared to children of the control group. The ratings of mothers with current diagnoses and mothers with no current diagnoses reached only marginal significance. Mothers with SCID diagnoses at the child's preschool age must have had at least one additional episode of depression and/or ongoing anxiety disorders, so we can assume that those women suffer more severely from symptoms than women with no current diagnoses. Due to psychiatric disorders mothers may experience their children as more difficult, more unadaptable, and more unpredictable compared to healthy ones, which could be due to a limitation of positive experiences [35].

Post hoc tests of the second mediation analysis regarding child responsiveness highlighted the crucial role of postpartum affective disorders for child behavior [12], as children of currently affected as well as not currently affected women displayed significantly less responsive behavior in interaction with their mothers than children in the control group. The results suggest that the motherchild interaction is impaired even if the mothers are not currently affected by psychiatric symptoms. This could be due to a lack of early positive experiences, leading to longterm consequences for the mother-child relationship.

Another explanation could be maternal attachment style insecurity, as mothers scoring high on the insecurity scale of the VASQ report constraints or fear of closeness and trusting others, which can lead to more avoidant behavior in interaction and hence to less maternal sensitivity [21]. Displayed child unresponsiveness in middle childhood has been linked to child insecure attachment [36]. Our findings could be a hint for the transmission of attachment insecurity from mother to child.

In sum, maternal mental health and maternal attachment insecurity can be seen as risk factors for the offspring. Referring to vulnerability-stress models, especially in critical developmental periods, these factors can increase the risk for the development of internalizing behavior in children. Internalizing behavior may not be apparent to others and, left untreated, may lead to internalizing disorders, and therefore prevention programs are urgently needed.

To shed further light on the relationship between internalizing child behavior, maternal attachment style in- 
security, and maternal mental health over time further research is clearly needed. The understanding of transmission pathways is of special importance for the implementation and evaluation of prevention and intervention programs.

\section{Limitations of the Study}

Besides a rather small sample size, our clinical sample consists of mothers with different anxiety disorders. Many mothers had more than one anxiety disorder. Furthermore, due to small subgroups and comorbidity of depression and anxiety or different anxiety disorders, it was not possible to identify specific mechanisms of distinct anxiety disorders. Our sample was characterized by an overproportion of academic degrees. Future studies should use larger and more homogeneous samples.

\section{Acknowledgments}

We thank the mothers and children who participated in the study as well as the maternity clinics and their staff in Heidelberg and Darmstadt for their cooperation and support in recruiting patients. The study was supported by a grant from the Program of Research Support of the Medical Faculty of Heidelberg University (funding period: 2003-2004) as well as by the Jacobs Foundation, Dietmar Hopp, and the Köhler and Jung Foundation.

\section{References}

1 Starr LR, Conway CC, Hammen CL, et al: Transdiagnostic and disorder-specific models of intergenerational transmission of internalizing pathology. Psychol Med 2014;44 161-172.

2 Achenbach TM, Rescorla LA: Manual for the ASEBA Preschool Forms and Profiles. Burlington, University of Vermont, Research Center for Children, Youth, and Families, 2000.

3 Biringen Z: The Emotional Availability (EA) Scales, ed 4. Unpublished Manual. Boulder, Colorado State University, 2008.

4 Martin SE, Clements ML, Crnic KA: Internalizing and externalizing symptoms in twoyear-olds: links to mother-toddler emotion processes. J Early Child Infant Psychol 2011; 7:105-128.

$>5$ Reck C, Struben K, Backenstrass M, et al: Prevalence, onset and comorbidity of postpartum anxiety and depressive disorders. Acta Psychiatr Scand 2008;118:459-468.

$\checkmark 6$ Newman DL, Moffitt TE, Caspi A, et al: Comorbid mental disorders: implications for treatment and sample selection. J Abnorm Psychol 1998;107:305-311.

7 Murray L, Cooper PJ: The impact of postpartum depression on child development. Int Rev Psychiatry 1996;8:55-63.

$>8$ Hammen C, Brennan PA: Severity, chronicity, and timing of maternal depression and risk for adolescent offspring diagnoses in a community sample. Arch Gen Psychiatry 2003;60: 253-258.

$\checkmark 9$ Hettema JM, Neale MC, Kendler KS: A review and meta-analysis of the genetic epidemiology of anxiety disorders. Am J Psychiatry 2001; 158:1568-1578

-10 Nomura Y, Warner V, Wickramaratne P. Parents concordant for major depressive disorder and the effect of psychopathology in offspring. Psychol Med 2001;31:1211-1222.
11 Goodman SH, Rouse MH, Connell AM, et al: Maternal depression and child psychopathology: a meta-analytic review. Clin Child Fam Psychol Rev 2011;14:1-27.

12 Field T, Healy B, Goldstein S, et al: Infants of depressed mothers show 'depressed' behavior even with nondepressed adults. Child Dev 1988;59:1569-1579.

13 Tronick E, Reck C: Infants of depressed mothers. Harv Rev Psychiatry 2009;17:147156.

14 Forehand R, Lautenschlager GJ, Faust J, et al: Parent perceptions and parent-child interactions in clinic-referred children: a preliminary investigation of the effects of maternal depressive moods. Behav Res Ther 1986;24: 73-75.

15 Zavos HMS, Rijsdijk FV, Eley TC: A longitudinal, genetically informative, study of associations between anxiety sensitivity, anxiety and depression. Behav Genet 2012;42:592602.

16 Richter L: The Importance of Caregiver-Child Interactions for the Survival and Healthy Development of Young Children: A Review. Geneva, World Health Organization, 2004

17 Cohn JF, Matias R, Tronick EZ, et al: Face-toface interactions of depressed mothers and their infants. New Dir Child Dev 1986;34:3145.

18 Feldman R, Greenbaum CW, Mayes LC, et al: Change in mother-infant interactive behavior: relations to change in the mother, the infant, and the social context. Infant Behav Dev 1997;20:151-163.

19 Field T, Hernandez-Reif M, Vera Y, et al: Anxiety and anger effects on depressed mother-infant spontaneous and imitative interactions. Infant Behav Dev 2005;28:1-9.

20 Vliegen N, Luyten P, Biringen Z: A multimethod perspective on emotional availability in the postpartum period. Parent Sci Pract 2009;9:228-243.
21 Ward MJ, Carlson EA: Associations among adult attachment representations, maternal sensitivity, and infant-mother attachment in a sample of adolescent mothers. Child Dev 1995;66:69-79.

22 McMahon C, Trapolini T, Barnett B: Maternal state of mind regarding attachment predicts persistence of postnatal depression in the preschool years. J Affect Disord 2008;107: 199-203.

23 Xue Y, Yang Y-P: Study on the adult attachment styles of the outpatients with anxiety disorder. Chin J Clin Psychol 2013;21:81-84.

24 Martins C, Gaffan EA: Effects of early maternal depression on patterns of infant-mother attachment: a meta-analytic investigation. J Child Psychol Psychiatry 2000;41:737-746.

-25 Biringen Z, Brown D, Donaldson L, et al: Adult Attachment Interview: linkages with dimensions of emotional availability for mothers and their pre-kindergarteners. Attach Hum Dev 2000;2:188-202.

26 O'Connor EE, Collins BA, Supplee L: Behavior problems in late childhood: the roles of early maternal attachment and teacher-child relationship trajectories. Attach Hum Dev 2012;14:265-288.

27 Najman JM, Williams GM, Nikles J, et al Mothers' mental illness and child behavior problems: cause-effect association or observation bias? J Am Acad Child Adolesc Psychiatry 2000;39:592-602.

-28 Ohrt T, Sjödin I, Thorell L-H: Cognitive distortions in panic disorder and major depression: specificity for depressed mood. Nord J Psychiatry 1999;53:459-464.

29 Richters JE: Depressed mothers as informants about their children: a critical review of the evidence for distortion. Psychol Bull 1992; 112:485-499. 
30 Wittchen H-U, Zaudig M, Fydrich T: SKID: Strukturiertes Klinisches Interview für DSMIV Achse I und II. Handanweisungen. Göttingen, Hogrefe, 1997.

31 Arbeitsgruppe Deutsche Child Behavior Checklist: Elternfragebogen für Klein- und Vorschulkinder (CBCL/1,5-5). Köln, Arbeitsgruppe Kinder, Jugend- und Familiendiagnostik (KJFD), 2000.

32 Arbeitsgruppe Deutsche Child Behavior Checklist: Fragebogen für ErzieherInnen von
Klein- und Vorschulkindern (C-TRF/1,5-5). Köln, Arbeitsgruppe Kinder, Jugend- und Familiendiagnostik (KJFD), 2000.

33 Bifulco A, Mahon J, Kwon JH, et al: The Vulnerable Attachment Style Questionnaire (VASQ): an interview-based measure of attachment styles that predict depressive disorder. Psychol Med 2003;33:1099-1110.

34 Zietlow A-L, Schlüter MK, Nonnenmacher N, et al: Maternal self-confidence postpartum and at pre-school age: the role of depression, anxiety disorders, maternal attachment insecurity. Matern Child Health J 2014;18:18731880.

35 Hopkins J, Campbell SB, Marcus M: Role of infant-related stressors in postpartum depression. J Abnorm Psychol 1987;96:237-241.

36 Easterbrooks MA, Biesecker G, Lyons-Ruth $\mathrm{K}$ : Infancy predictors of emotional availability in middle childhood: the roles of attachment security and maternal depressive symptomatology. Attach Hum Dev 2000;2:170-187. 